\title{
On the mobility degree of (pseudo-) Riemannian spaces with respect to concircular mappings
}

\author{
Olena Chepurna and Irena Hinterleitner
}




\title{
ON THE MOBILITY DEGREE OF (PSEUDO-) RIEMANNIAN SPACES WITH RESPECT TO CONCIRCULAR MAPPINGS
}

\author{
OLENA CHEPURNA AND IRENA HINTERLEITNER
}

\begin{abstract}
In this paper we study the mobility degree of (pseudo-) Riemannian spaces with respect to concircular mappings. We assume that the smoothness class of differentiability is $C^{2}$.

2000 Mathematics Subject Classification: 53B20, 53B21, 53B30, 53C26

Keywords: geodesic mappings, (pseudo-)Riemannian manifold, smoothness class
\end{abstract}

\section{INTRODUCTION}

Under a geodesic circle we understand a curve for which the first curvature is constant and the second curvature is zero. K. Yano [14] introduced a conformal mapping of (pseudo-) Riemannian spaces which preserves geodesic circles and is called concircular.

These mappings are studied in many papers. In the present paper, we show results connected with basic notations under the conditions of minimal differentiability of metrics and geometric objects which define concircular mappings and also concircular vector fields.

\section{FUNDAMENTAL EQUATIONS OF CONCIRCULAR MAPPINGS}

Let $V_{n}=(M, g)$ and $\bar{V}_{n}=(\bar{M}, \bar{g})$ be $n$-dimensional (pseudo-) Riemannian manifolds with the metric tensors $g$ and $\bar{g}$, respectively, $n>2$.

Definition 1. A conformal mapping is a diffeomorphism of $V_{n}$ onto $\bar{V}_{n}$ such that for all points $x \in M(\equiv \bar{M})$ the following relation is satisfied

$$
\bar{g}(x)=e^{2 \sigma(x)} g(x),
$$

where $\sigma$ is a function on $M$.

If $\sigma$ is constant, then the mapping is homothetic, and, moreower, if $\sigma=0$, then the mapping is isometric. See $[1,7,9,10,12]$.

The paper was supported by the project FAST-S-12-25/1660 of the Brno University of Technology. 
As we have checked (see [14], [9, p. 117]), if a pseudo-Riemannian space admits concircular mappings, then the function of conformality $\vartheta \stackrel{\text { def }}{=} \mathrm{e}^{-\sigma}$ satisfies

$$
\nabla \nabla \vartheta=\rho \cdot g,
$$

where $\rho$ is a function and $\nabla$ is the Levi-Civita connection with respect to the metric $g$. In a local coordinate neighbourhood $(U, x), U \subset M$, it has the form $\nabla_{i} \vartheta_{j}=\rho g_{i j}$, where $g_{i j}$ are components of $g$ and $\vartheta_{j}=\nabla_{j} \vartheta$. A vector field $\vartheta_{i}$ is called equidistant (Sinyukov [12, p. 92], see [9, p. 82]).

The integrability conditions of the last set of equations read

$$
\vartheta_{\alpha} R_{i j k}^{\alpha}=g_{i j} \nabla_{k} \rho-g_{i k} \nabla_{j} \rho,
$$

where $R_{i j k}^{h}$ are components of the Riemann tensor of $V_{n}$. Using contraction, we get:

$$
\nabla_{i} \rho=-\frac{1}{n-1} \vartheta_{\alpha} R_{i}^{\alpha}
$$

where $R_{i}^{h}=g^{h \alpha} R_{\alpha i}$ and $R_{i j}=R_{i \alpha j}^{\alpha}$ are components of the Ricci tensor on $V_{n}$.

Remark 1. In many papers, the Ricci tensor was defined with the opposite sign, for example, $[2-8,12]$.

Contracting the integrability condition (2.3) with $g^{i \beta} \vartheta_{\beta}$, we obtain easily $\nabla_{k} \rho=$ $B \vartheta_{k}$, where $B$ is a function. Because $\vartheta_{k}$ is gradient-like: $\vartheta_{k}=\nabla_{k} \vartheta$, then it implies that $\rho=\rho(\vartheta)$ and $B=B(\vartheta)$.

After this, the condition (2.3) acquires the following form

$$
\vartheta_{\alpha} R_{i j k}^{\alpha}=B\left(g_{i j} \vartheta_{k}-g_{i k} \vartheta_{j}\right) .
$$

As was shown earlier [13] (see [3,4,6,9]), these equations are satisfied if

$$
V_{n}, \bar{V}_{n} \in C^{2} \text { (i.e. } g_{i j}(x), \bar{g}_{i j}(x) \in C^{2} \text { ), } \vartheta(x) \in C^{3}, \vartheta_{i}(x) \in C^{2} \text { and } \varrho(x) \in C^{1} \text {. }
$$

\section{FUNDAMENTAL EQUATIONS OF CONCIRCULAR MAPPINGS FOR MINIMAL DIFFERENTIABLE CONDITIONS}

We can write formula (2.2) in the following form

$$
\nabla_{j} \vartheta^{i} \equiv \frac{\partial \vartheta^{i}}{\partial x^{j}}+\Gamma_{\alpha j}^{i} \vartheta^{\alpha}=\varrho \cdot \delta_{j}^{i},
$$

where $\vartheta^{i}=g^{i \alpha} \vartheta_{\alpha}, \delta_{j}^{i}$ is the Kronecker symbol and $\Gamma_{i j}^{h}$ are the Christoffel symbols. It is easily seen that formulas (3.1) and also (2.2) are true when

$$
\left.V_{n}, \bar{V}_{n} \in C^{1} \text { (i.e. } g_{i j}(x), \bar{g}_{i j}(x) \in C^{1}\right), \vartheta(x) \in C^{2}, \vartheta_{i}(x) \in C^{1} \text { and } \varrho(x) \in C^{0} \text {. }
$$

The following lemma holds. 
Lemma 1 (Hinterleitner and Mikeš [2]). Let $\lambda^{h} \in C^{1}$ be a vector field and $\rho$ a function. If

$$
\frac{\partial \lambda^{h}}{\partial x^{i}}-\rho \delta_{i}^{h} \in C^{1}
$$

then $\lambda^{h} \in C^{2}$ and $\rho \in C^{1}$.

If $\Gamma_{i j}^{h} \in C^{1}$ holds, which is equivalent to $V_{n} \in C^{2}$ (i.e., $g_{i j} \in C^{2}$ ), then from formula (3.1) follows $\frac{\partial \vartheta^{i}}{\partial x^{j}}-\varrho \cdot \delta_{j}^{i} \in C^{1}$, and from Lemma 1 we get:

$$
\vartheta^{i}(x) \in C^{2}\left(\equiv \vartheta_{i}(x) \in C^{2} \equiv \vartheta(x) \in C^{3}\right) \text { and } \varrho(x) \in C^{1} .
$$

From this viewpoint, we specify and generalize the results involving concircular vector fields below. Evidently, in this case, the above formulas from (2.3) to (2.5) are satisfied.

The system of equations

$$
\begin{aligned}
\nabla_{i} \vartheta_{j} & =\rho \cdot g_{i j}, \\
\nabla_{i} \rho & =-\frac{1}{n-1} \vartheta_{\alpha} R_{i}^{\alpha}
\end{aligned}
$$

is closed. It is a system of linear differential equations with respect to the co-vector $\vartheta_{i}$ and function $\varrho$, of Cauchy type, in first order covariant derivatives with coefficients uniquely determined by the metric $g$ of the (pseudo-) Riemannian space $V_{n}$. For any family of initial values $\vartheta_{i}\left(x_{0}\right)=\vartheta_{i}^{\circ}$ and $\rho\left(x_{0}\right)=\rho^{\circ}$ of the functions under consideration in the given point $x_{0}$, it admits at most one solution. Consequently, the number of free parameters in the general solution of the system is at most $n+1$. See $[6,13]$.

Definition 2. The upper bound for the number of substantial parameters in the general solution of the system of equations (2.2) is called the mobility degree under concircular mappings of the (pseudo-) Riemannian manifold $V_{n}$.

Since the system is linear, it admits at most $n+1$ linearly independent solutions corresponding to constant coefficients. It is obvious that the mobility degree under concircular mappings of the space coincides with the cardinality of the system of independent (substantial) concircular vector fields of the space.

It is known that only spaces with constant curvature admit the maximal number of $n+1$ linearly independent concircular vector fields. Hence, under concircular mappings, only the spaces of constant curvature have the maximal mobility degree. This holds locally.

It follows from the analysis of the system of equations (3.2) that if $V_{n} \in C^{r}, r \geq 2$, then $\vartheta_{i} \in C^{r}$ and $\rho \in C^{r-1}$. It follows that the function $\vartheta$ belongs to $C^{r+1}$. From this and the formula (2.1), we obtain the following theorem. 
Theorem 1. If the (pseudo-) Riemannian manifold $V_{n}\left(V_{n} \in C^{r}, r \geq 2, n>2\right)$ admits a concircular mapping onto $\bar{V}_{n} \in C^{2}$, then $\bar{V}_{n}$ belongs to $C^{r}$. Moreover, the function $\vartheta$ of conformality $V_{n}$ and $\bar{V}_{n}: \bar{g}=\vartheta^{-2} \cdot g$ belongs to $C^{r+1}$.

We suppose that the differentiability class $r$ is equal to $2,3, \ldots, \infty, \omega$, where $\infty$ and $\omega$ denote infinitely differentiable and real analytic functions, respectively.

We can construct examples of such concircular mappings $V_{n} \rightarrow \bar{V}_{n}$ in the form of equidistant metrics, see [9, p. 79]:

$$
\bar{g}=\frac{1}{\vartheta^{2}} \cdot g, \quad g= \pm\left(d x^{1}\right)^{2}+\text { const } \cdot \sqrt{\left|\vartheta^{\prime}\right|} \cdot d \tilde{s}^{2},
$$

where $d \tilde{s}^{2}\left(x^{2}, \ldots, x^{n}\right)$ is a $C^{r}$ metric of an $(n-1)$-dimensional (pseudo-) Riemannian space $\tilde{V}_{n-1}$ and $\vartheta\left(x^{1}\right)$ is a $C^{r+1}$ function and $\vartheta>0, \vartheta^{\prime} \neq 0$.

\section{A (PSEUDO-) RIEMANNIAN SPACE WHICH ADMITS AT LEAST TWO LINEARLY INDEPENDENT CONCIRCULAR VECTOR FIELDS}

Below we prove the following properties of concircular fields.

Lemma 2. The non-vanishing concircular vector field $\vartheta_{i}(x)$ can be equal to zero only on point sets of zero measure.

Proof. Let us suppose that Lemma 2 is not true. Thus there exists a point $x_{0} \in$ $M$ in the neighborhood $U_{x_{0}} \subset M$ of which the concircular vector field $\vartheta_{i}(x)$ is vanishing. From (3.2) follows that $\rho(x)=0$ on $U_{x_{0}}$. From that follows the initial conditions at the point $x_{0}: \vartheta_{i}\left(x_{0}\right)=0$ and $\rho\left(x_{0}\right)=0$. The system of linear equations (3.2) with these initial conditions has only the trivial solution $\vartheta_{i}(x)=0$ and $\rho(x)=$ 0 on all of $M$.

By mathematical induction we have the following lemma.

Lemma 3. The set of $r(r<n)$ linear independent concircular vector fields

$$
\left\{\begin{array}{l}
1 \\
\left\{\vartheta_{i}, \stackrel{\vartheta}{\vartheta}_{i}, \ldots, \stackrel{r}{\vartheta}_{i}\right.
\end{array}\right\}
$$

on $V_{n}$ can be linearly dependent only on point sets of zero measure.

Proof. Successively we are able to substitute $r=1,2, \ldots, n-1$. Let (4.1) be linearly independent (excluding at point sets of zero measure) concircular vector fields on $V_{n}$ which satisfy the equations

$$
\stackrel{s}{\vartheta}_{i, j}={\stackrel{s}{\rho} g_{i j}}
$$

where $\stackrel{s}{\rho}$ are functions on $V_{n}$.

Let these vectors be linearly independent at the point $x_{0} \in M$, then these are linearly independent at a point $x$ in a certain neighborhood $U_{x_{0}}$. Finally, let $\vartheta_{i}$ be a concircular vector field on $M$ and

$$
\vartheta_{i}(x)=\sum_{s=1}^{r} \stackrel{s}{\alpha}(x) \cdot \stackrel{s}{\vartheta}_{i}(x) \text { for } x \in U_{x_{0}}
$$


where $\stackrel{s}{\alpha}(x)$ are functions on $U_{x_{o}}$. Because $\stackrel{s}{\vartheta}_{i}(x) \in C^{1}$, the functions $\stackrel{s}{\alpha}(x)$ are differentiable. Covariantly differentiating (4.2) with respect to $x^{j}$ we find

$$
\left(\rho-\sum_{s=1}^{r} \stackrel{s}{\alpha} \cdot \stackrel{s}{\rho}\right) g_{i j}=\sum_{s=1}^{r} \nabla_{j} \stackrel{s}{\alpha} \cdot \stackrel{s}{\vartheta}_{i}
$$

This implies that $\rho=\sum_{s=1}^{r} \stackrel{s}{\alpha} \cdot \stackrel{s}{\rho}$ and $\nabla_{j} \stackrel{s}{\alpha}=0$ (i. e., $\stackrel{s}{\alpha}=$ const) on $U_{x_{o}}$.

For the initial conditions

$$
\begin{gathered}
\vartheta_{i}\left(x_{o}\right)=\sum_{s=1}^{r} \stackrel{s}{\alpha} \cdot \stackrel{s}{\vartheta}_{i}\left(x_{o}\right), \\
\rho\left(x_{o}\right)=\sum_{s=1}^{r} \stackrel{r}{\alpha} \cdot \stackrel{r}{\rho}\left(x_{o}\right),
\end{gathered}
$$

the equations (3.2) have only one solution: $\vartheta_{i}(x)=\sum_{s=1}^{r} \stackrel{s}{\alpha} \cdot \stackrel{s}{\vartheta_{i}}(x)$ on $V_{n}$.

We are going to prove the following

Theorem 2. If a (pseudo-) Riemannian space $V_{n} \in C^{2}(n>2)$ admits at least two linearly independent concircular vector fields $\vartheta_{i}(x) \in C^{1}$ with constant coefficients, then $B$ is a constant, uniquely determined by the metric of the space $V_{n}$.

Remark 2. In [6] and [4, p. 88] a similar theorem was published, but the proof was done only for $V_{n} \in C^{3}, \vartheta_{i}(x) \in C^{3}$ and $\varrho(x) \in C^{2}$, and, moreover, it has local validity. This also concerns the following Theorems 3, 4 and 5. On the basis of Lemmas 2 and 3 these Theorems are valid globally.

Proof. Assume in $V_{n}$ exist at least two linearly independent concircular vector fields with constant coefficients $\vartheta_{i}$ and $\tilde{\vartheta}_{i}$, with correspondent functions $B$ and $\tilde{B}$, respectively. Then the following is satisfied (see (3.1)):

$$
\begin{aligned}
& \vartheta_{\alpha} R_{i j k}^{\alpha}=B\left(g_{i j} \vartheta_{k}-g_{i k} \vartheta_{j}\right), \\
& \tilde{\vartheta}_{\alpha} R_{i j k}^{\alpha}=\tilde{B}\left(g_{i j} \tilde{\vartheta}_{k}-g_{i k} \tilde{\vartheta}_{j}\right) .
\end{aligned}
$$

Multiplying (4.3) by $\tilde{\vartheta}_{\alpha} g^{\alpha k}$ and contracting over $k$ we get by (4.4)

$$
(B-\tilde{B})\left(g_{i j} \vartheta_{\alpha} \tilde{\vartheta}^{\alpha}-\tilde{\vartheta}_{i} \vartheta_{j}\right)=0 .
$$

Suppose $B \neq \tilde{B}$. Then $g_{i j} \vartheta_{\alpha} \tilde{\vartheta}^{\alpha}-\tilde{\vartheta}_{i} \vartheta_{j}=0$. From the last formula we get $\vartheta_{\alpha} \tilde{\vartheta}^{\alpha}=0$ and $\tilde{\vartheta}_{i} \vartheta_{j}=0$, a contradiction, since the vector fields are non-zero.

Hence $B=\tilde{B}$ holds. That is, the function $B$ is uniquely defined by the metric of the space $V_{n}$ itself. Because $\vartheta_{k}$ and $\tilde{\vartheta}_{k}$ are gradient-like covector fields $\left(\vartheta_{k}=\nabla_{k} \vartheta\right.$ and $\left.\tilde{\vartheta}_{k}=\nabla_{k} \tilde{\vartheta}\right)$ from the equality $B=\tilde{B}$ the fact $B(\vartheta)=\tilde{B}(\tilde{\vartheta})$ follows. Note that $\vartheta$ and $\tilde{\vartheta}$ are indenpendent variables, then from this fact follows: $B$ is constant. 
Note that the above theorem is analogous to some results proven earlier under the additional assumptions $V_{n}, \bar{V}_{n} \in C^{3},[5,6,13]$.

Theorem 3. There are no (pseudo-) Riemannian spaces $V_{n} \in C^{2}$, except spaces of constant curvature, which admit more than $(n-2)$ linearly independent concircular vector fields $\vartheta_{i}(x) \in C^{1}$ corresponding to constant coefficients.

Remark 3. In [4, p. 86], [3,5], a similar theorem was published but the proof was done only for $V_{n} \in C^{3}, \vartheta_{i}(x) \in C^{3}$ and $\varrho_{i}(x) \in C^{2}$.

Proof. Let us suppose the opposite. Let $V_{n}$ be a space which is not of constant curvature and yet admits more than $(n-2)$ linearly independent concirrcular vector fields with constant coefficients. The conditions (2.5) read

$$
\vartheta_{\alpha} Z_{i j k}^{\alpha}=0
$$

where

$$
Z_{i j k}^{h} \stackrel{\text { def }}{=} R_{i j k}^{h}-B\left(\delta_{k}^{h} g_{i j}-\delta_{j}^{h} g_{i k}\right)
$$

We can write the tensor $Z_{i j k}^{h}$ as

$$
Z_{i j k}^{h}=\sum_{s=1}^{m} b_{s}^{h} \stackrel{s}{\Omega}_{i j k},
$$

where $b_{s}{ }^{h}$ are some linearly independent vectors, and $\stackrel{s}{\Omega}_{i j k}$ are linearly independent tensors. Since $V_{n}$ is not of constant curvature, $m \geq 2$ holds.

From the conditions (4.5), we obtain

$$
\vartheta_{\alpha} \underset{1}{b^{\alpha}}=0, \quad \vartheta_{\alpha} \underset{2}{b^{\alpha}}=0, \quad \cdots \quad, \vartheta_{\alpha}{\underset{m}{b}}^{\alpha}=0 .
$$

Since $m \geq 2$, among the equations of the system (4.6) there are at least two substantial equations. From the previous facts it follows that there exist less or equal to $n-2$ linearly independent vector fields $\vartheta_{i}$, a contradiction. This proves Theorem 3 .

From Theorem 3 and results in [6], the following two theorems are obtained:

Theorem 4. Let $V_{n} \in C^{2},(n>2)$, be (pseudo-) Riemannian spaces in which there are $(n-2)$ linearly independent concircular vector fields $\vartheta_{i}(x) \in C^{1}$. Then the Riemannian tensor has the following expression

$$
R_{h i j k}=B\left(g_{h k} g_{i j}-g_{h j} g_{i k}\right)+e\left(a_{h} b_{i}-a_{i} b_{h}\right)\left(a_{j} b_{k}-a_{k} b_{j}\right),
$$

where $a_{i}$ and $b_{i}$ are non-colinear and pairwise orthogonal covectors, $e= \pm 1$, and $B=$ const. 
Theorem 5. The (pseudo-) Riemannian space $V_{n} \in C^{3}(n>3)$ admits $(n-2)$ linearly independent concircular vector fields $\vartheta_{i}(x) \in C^{1}$ if and only if in $V_{n}$ the relations [11]

$$
\begin{aligned}
R_{h i j k} & =B\left(g_{h k} g_{i j}-g_{h j} g_{i k}\right)+e\left(a_{h} b_{i}-a_{i} b_{h}\right)\left(a_{j} b_{k}-a_{k} b_{j}\right), \\
a_{i, j} & =\stackrel{1}{\xi}_{j} a_{i}+\stackrel{2}{\xi}_{j} b_{i}+c_{i} a_{j} \\
b_{i, j} & =\stackrel{3}{\xi}_{j} a_{i}+\stackrel{4}{\xi}_{j} b_{i}+c_{i} b_{j} \\
c_{i, j} & =\stackrel{5}{\xi_{j}} a_{i}+\stackrel{6}{\xi_{j}} b_{i}+c_{i} c_{j}-B g_{i j}
\end{aligned}
$$

are satisfied, where $a_{i}$ and $b_{i}$ are non-colinear and pairwise orthogonal covectors; $c_{i}, \stackrel{s}{\xi_{j}}(s=1, \ldots, 6)$ are some covectors; $e= \pm 1$, and $B=$ const.

Remark. This theorem was proved locally for $V_{n} \in C^{3}, \vartheta_{i} \in C^{3}, \varrho \in C^{2}$, in [6]. The detailed local proof is contained in the dissertation [3, p. 94-95], [4, p. 88-92].

\section{REFERENCES}

[1] L. P. Eisenhart, Riemannian geometry. Princeton Univ. Press,, 1949.

[2] I. Hinterleitner and J. Mikeš, "Geodesic mappings and einstein spaces," in Geometric Methods in Physics, vol. 19, 2013, pp. 331-336.

[3] V. A. Kiosak, "On geodesic mappings of Riemannian spaces," Ph.D. dissertation, Mechnikov Univ. Odessa, 1993.

[4] V. A. Kiosak, "On geodesic mappings of Riemannian spaces," Ph.D. dissertation, Palacky Univ. Olomouc, 2002.

[5] V. A. Kiosak, "On equidistant pseudo-riemannian spaces," Mat. Stud., vol. 36, pp. 21-25, 2011.

[6] V. A. Kiosak and J. Mikeš, "On the degree of mobility of Riemannian spaces with respect to geodesic mappings," in Geometriya Pogruzh. Mnogoobraz., Moscow, 1986, pp. 35-39.

[7] J. Mikeš, "Geodesic mappings of affine-connected and Riemannian spaces," J. Math. Sci., vol. 78, pp. 311-333, 1996.

[8] J. Mikeš, "Holomorphically projective mappings and their generalizations," J. Math. Sci., vol. 89, no. 3, pp. 1334-1353, 1998.

[9] J. Mikeš, A. Vanžurová, and I. Hinterleitner, Geodesic mappings and some generalizations. Olomouc: Palacky University Press, 2009.

[10] A. Z. Petrov, New methods in the general theory of relativity. Moscow: Nauka, 1966.

[11] Z. Radulovich, J. Mikeš, and M. L. Gavril'chenko, Geodesic mappings and deformations of Riemannian spaces. C CID, Podgorica, OGU, Odessa, 1997.

[12] N. S. Sinyukov, Geodesic mappings of Riemannian spaces. Moscow: Nauka, 1979.

[13] H. L. Vries, "Über riemannsche Raume die infinitesimale konforme Transformationen gestätten," math. Zeitschr., vol. 60, no. 3, pp. 328-347, 1954.

[14] K. Yano, Differential geometry on complex and almost complex spaces. Pergamon press, 1965. 
Authors' addresses

Olena Chepurna

Odessa State Economical University, Department of Mathematics, Odessa, Ukraine

E-mail address: culeshova@ukr. net

Irena Hinterleitner

Brno University of Technology, Faculty of Civil Engineering, Department of Mathematics, Žižkova 17, 602 00, Brno, Czech Republic

E-mail address: hinterleitner.irena@seznam.cz 